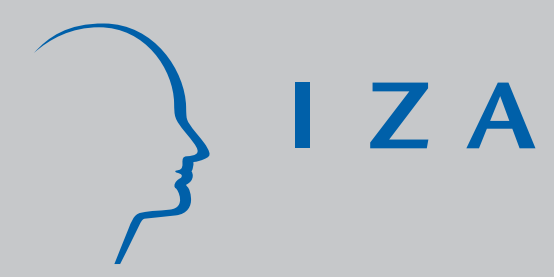

IZADP No. 2244

Wirtschaftliche Entwicklung und Demokratie: Ist Demokratie ein Wohlstandsmotor oder ein Wohlstandsprodukt?

Uwe Sunde

August 2006 


\title{
Wirtschaftliche Entwicklung und Demokratie: Ist Demokratie ein Wohlstandsmotor oder ein Wohlstandsprodukt?
}

\author{
Uwe Sunde \\ IZA Bonn \\ and University of Bonn
}
Discussion Paper No. 2244
August 2006

IZA

P.O. Box 7240

53072 Bonn

Germany
Phone: +49-228-3894-0
Fax: +49-228-3894-180
Email: iza@iza.org

\begin{abstract}
Any opinions expressed here are those of the author(s) and not those of the institute. Research disseminated by IZA may include views on policy, but the institute itself takes no institutional policy positions.

The Institute for the Study of Labor (IZA) in Bonn is a local and virtual international research center and a place of communication between science, politics and business. IZA is an independent nonprofit company supported by Deutsche Post World Net. The center is associated with the University of Bonn and offers a stimulating research environment through its research networks, research support, and visitors and doctoral programs. IZA engages in (i) original and internationally competitive research in all fields of labor economics, (ii) development of policy concepts, and (iii) dissemination of research results and concepts to the interested public.
\end{abstract}

IZA Discussion Papers often represent preliminary work and are circulated to encourage discussion. Citation of such a paper should account for its provisional character. A revised version may be available directly from the author. 


\section{ABSTRACT \\ Wirtschaftliche Entwicklung und Demokratie: Ist Demokratie ein Wohlstandsmotor oder ein Wohlstandsprodukt?}

Praktisch alle wirtschaftlich entwickelten Länder der Welt sind demokratisch. Sind demokratische Strukturen also kausal für wirtschaftlichen Wohlstand und Wachstum? Oder ist es vielmehr der wirtschaftliche Entwicklungsstand eines Landes, der eine Demokratie erst ermöglicht? Dieser Artikel gibt einen Überblick über die jüngere empirische Literatur zur Frage der Kausalität hinter der positiven Korrelation zwischen der Wahrscheinlichkeit demokratischer Strukturen und wirtschaftlichem Wohlstand und Wachstum. Die Evidenz lässt Zweifel an einem direkten kausalen Effekt in irgendeiner Richtung aufkommen. Allerdings deuten die Ergebnisse auf indirekte Effekte hin. So schaffen Demokratien offensichtlich bessere Rahmenbedingungen für die Akkumulation von Humankapital, insbesondere durch die Gewährleistung eines Rechtsstaats, und somit für wirtschaftliche Entwicklung. Andererseits scheint nicht Wohlstand an sich, sondern ein damit einhergehendes geeignetes gesellschaftliches Umfeld, wie etwa geringe Ungleichheit, demokratische Strukturen erst zu ermöglichen.

Economically highly developed countries are mostly democratic. But does this association constitute a causal relationship according to which democracy is a determinant of economic development? Or is it, conversely, economic development that paves the way for democratization? This paper gives an overview of the recent empirical literature that has dealt with this question. The empirical evidence raises doubts about the existence of any direct causation. However, there seem to be indirect causal mechanisms. Democracies seem to implement better conditions for the accumulation of human capital, in particular in terms of a rule of law. On the other hand do democracies not simply arise as consequence of economic development, but because of an adequate social environment with little inequality, that may be associated with economic well-being.

JEL Classification: $\quad \mathrm{H} 10, \mathrm{~N} 10, \mathrm{O} 10, \mathrm{E} 60$

Keywords: democracy, development, Lipset hypothesis, causal effect, growth, political institutions

Corresponding author:

Uwe Sunde

IZA

Schaumburg-Lippe Str. 7-9

D-53072 Bonn

Germany

E-mail: sunde@iza.org

\footnotetext{
* Der Autor dankt Julia Kretzschmar für tatkräftige Unterstützung bei der Literaturrecherche, sowie Martina Ermisch, Lars P. Feld und einem Gutachter für sehr hilfreiche Hinweise und Kommentare.
} 


\section{Einleitung und methodische Vorbemerkungen}

Nach einer weit verbreiteten Ansicht ist die Demokratie ein Wohlstandsmotor. Ein flüchtiger Blick auf die Welt scheint diese Sicht zu bestätigen: Die meisten reichen Staaten werden demokratisch, die meisten armen Staaten autoritär regiert. Diese Korrelation lässt jedoch genauso den umgekehrten Schluss zu, nämlich dass demokratische Strukturen das Ergebnis wirtschaftlichen Wohlstands sind. Angesichts der Konflikte im Nahen Osten und in Südamerika erhält die Frage nach einem Kausalzusammenhang zwischen Demokratie und wirtschaftlichem Wohlstand eine besondere Aktualität: Ist die Demokratie notwendige Voraussetzung für den Wohlstand oder verhält es sich umgekehrt so, dass Wohlstand notwendige Voraussetzung für Demokratie ist? Die praktische Relevanz dieser Frage für die Außen- und Entwicklungspolitik ist offenkundig. Die meisten Industrieländer haben zwar sowohl die wirtschaftliche Entwicklung als auch die Demokratisierung der Staaten der so genannten Dritten Welt als Ziele ihrer Entwicklungspolitik festgeschrieben, jedoch herrscht Uneinigkeit im Hinblick auf die Priorität dieser Ziele und ihre konkrete Umsetzung in praktische Politik. Lange Zeit wurden Diktaturen geduldet und gestützt, um die wirtschaftliche Entwicklung und auf lange Sicht eine Demokratisierung zu ermöglichen. In anderen Zusammenhängen, beispielsweise im Fall des Irakkonflikts, wird dagegen der Demokratisierung in der praktischen Außenpolitik Vorrang eingeräumt, um damit eine Befriedung herbeizuführen und somit ein entwicklungsfähiges Umfeld zu schaffen.

Über die Frage nach dem kausalen Zusammenhang zwischen Demokratie und wirtschaftlicher Entwicklung ist auch in der ökonomischen Literatur eine Diskussion entbrannt. Dabei beschäftigen sich die Vertreter der neuen institutionellen Sichtweise mit der Frage, ob und wie demokratische Strukturen die wirtschaftliche Entwicklung beeinflussen können. Demokratie, so ihr Argument, kann direkt oder mittelbar über die durch demokratische Institutionen implementierten Politikmaßnahmen, wirtschaftlichen Institutionen oder Anreize den Wohlstand vermehren. Konsequenz dieses Ansatzes ist, dass der Implementierung demokratischer Strukturen Priorität einzuräumen ist, um eine geeignete Basis für die wirtschaftliche Entwicklung zu schaffen. Nach der alternativen Sichtweise des "Entwicklungsansatzes" der Modernisierungstheorie (Lipset, 1959) ist die Demokratisierung selbst eine Funktion des sozioökonomischen Entwicklungsstandes. Wenn die Modernisierung des politischen und wirtschaftlichen Systems nicht gleichzeitig möglich ist, so muss nach dieser Sichtweise der wirtschaftlichen Entwicklung Vorrang eingeräumt werden. Eine direkte Implikation aus dieser Theorie, die lange Zeit die Realität in der internationalen Politik bestimmte, ist, dass unter Umständen Diktaturen temporär unterstützt werden müssen, um schließlich zur Demokratie gelangen zu können. 
Die Frage, ob die Korrelation zwischen Wohlstand und Demokratie einen Kausalzusammenhang in einer bestimmten Richtung impliziert, kann letztendlich nur empirisch beantwortet werden. Die vorliegende Arbeit soll einen Überblick über die neuere empirische Literatur und deren Ergebnisse $\mathrm{zu}$ dieser Frage geben. Aufgrund des komplexen Untersuchungsgegenstandes kann dieser Artikel keinen erschöpfenden Überblick über die Literatur geben. Vielmehr wird das Augenmerk auf zeitgenössische Beiträge gelegt, die direkte kausale Effekte zu identifizieren versuchen. Frühe Studien, die mit Hilfe von Querschnittsdaten eine Korrelation etablierten und diese als Kausalbeziehung in eine bestimmte Richtung interpretierten, weisen zwei zentrale Schwächen auf, die die Ergebnisse möglicherweise verfälschen. Zum einen ist es mit Querschnittsdaten schwierig, umgekehrte Kausalität (reverse causality) auszuschließen, es sei denn man hat triftige Gründe anzunehmen, die Variation in der als treibend angenommenen Variablen als modellexogen $\mathrm{zu}$ betrachten. Im Fall des politischen Systems und des Wohlstandes dürfte es jedoch problematisch sein, in diese Richtung zu argumentieren. Zum anderen ist das zentrale Identifikationsproblem im gegebenen Zusammenhang durch die Datenlage erschwert, da Variation über eine überschaubare Anzahl an Ländern genutzt werden muss. Dies vergrößert die Gefahr, dass eine relevante Bestimmungsgröße nicht beobachtbar ist, oder nicht in die Schätzung aufgenommen werden kann, um das Modell nicht zu überladen. Bekanntlich führt die daraus resultierende Auslassung relevanter Variablen zu einer systematischen Verzerrung der Schätzergebnisse. Die neuere Literatur, auf die sich der vorliegende Artikel hauptsächlich konzentriert, verwendet daher Längsschnittsdaten (Panel-Daten), um die Effekte von Interesse über die zeitliche Variation zu identifizieren und von zeitinvarianten länderspezifischen Einflussfaktoren zu trennen. Darüber hinaus werden vermehrt Instrumentvariablenschätzer verwendet, die auf theoretischen Zusammenhängen und Wirkungskanälen basieren und gleichzeitig modellexogene Variation ausnutzen um Endogenitätsprobleme zu vermeiden.

Die Untersuchung des empirischen Zusammenhangs zwischen Demokratie einerseits und wirtschaftlichem Wohlstand andererseits erfordert eine grundlegende Bestimmung der Begriffe und entsprechenden empirischen Maße. Die fundamentalste und wortgetreuste Definition von Demokratie kommt aus dem Griechischen, von demos "Volk" und kratein "herrschen" und bezeichnet damit eine Staatsform, in der die Staatsgewalt vom Volk ausgeht. Darüber, was Demokratie genau ausmacht, herrschen jedoch unterschiedliche Vorstellungen in der Politikwissenschaft. ${ }^{1}$ Eine mögliche Definition von Demokratie im engeren Sinne besteht in der

\footnotetext{
${ }^{1}$ Mazo (2005) bietet einen Überblick von Bedingungen, die für eine demokratische Struktur gelten müssen. Das Spektrum reicht von minimalistischen Standards in der Tradition von Schumpeter, Przeworksi und Huntington, die mit Demokratie in erster Linie Wahlen verbinden, bis hin zu maximalen Forderungen von post-modernen Theoretikern und auch Feministen, denen es vor allem auf politische, und letztendlich, kollektive Freiheit ankommt. Siehe auch Lijphart (1999).
} 
Existenz (bzw. Abwesenheit) von Möglichkeiten zur politischen Mitwirkung und dem freien Wettbewerb zwischen politischen Positionen, siehe Dahl (1971). Diese Definition soll bei der Diskussion der empirischen Arbeiten als Grundlage dienen. Das Problem der Bestimmung, was letztlich eine Demokratie ausmacht überträgt sich auf die Frage nach einer sinnvollen und köhärenten empirischen Messung von Demokratie - auch insoweit gibt es alternative Ansätze in der Literatur. Dabei werden Demokratien meistens anhand von Indizes identifiziert, die die politischen Rechte und bürgerliche Freiheiten in einem Staatsgebilde evaluieren und quantifizieren. Obwohl diese Demokratieindizes ähnliche Charakteristika aufweisen, unterscheiden sie sich in ihren Skalen und deren Interpretierbarkeit. ${ }^{2}$ In der empirischen Praxis werden Demokratieindizes vergröbert oder gebündelt, um Messfehler und Probleme bei der Vergleichbarkeit zu vermeiden. Vorherrschend sind dabei die Erfassung von Demokratie als bivariate Variable (demokratisch-undemokratisch), siehe z.B. Przeworski und Limongi (1997), oder durch synthetische zusammengefasste Indikatoren, die den Grad an Demokratie oder der Qualität der demokratischen Institutionen widerspiegeln sollen (siehe bspw. Burkhart und LewisBeck, 1994, oder den Polity IV-Index). ${ }^{3}$ Zum Teil reflektieren diese Indizes auch einen Demokratiebegriff in einem weiteren Sinne, der über Wahlrechte und Minderheitenschutz hinaus auch Gewaltenteilung und Rechtsstaatlichkeit beinhaltet. Für ein detailliertes Verständnis der Kausalzusammenhänge und Mechanismen ist es jedoch nötig, die Defintion von Demokratie im engeren Sinne als Staatsform mit Wahl- und Gestaltungsrechten für alle Bürger von damit verbundenen, aber doch unterschiedlichen Institutionen wie etwa dem Rechtsstaat zu trennen.

Denn im Gegensatz zum Demokratiebegriff, der sich auf die politische Mitwirkung aller Gesellschaftsmitglieder bezieht, sichert der Rechtsstaat allen Bürgern Grundrechte und Gleichheit vor dem Gesetz zu. Dieses Grundprinzip der Isonomie impliziert sowohl Rechte, insbesondere Eigentums- und Persönlichkeitsrechte des Einzelnen gegenüber der Staatsmacht, als auch Schutz im Verkehr mit anderen Bürgern im privatrechtlichen Sinne. ${ }^{4}$ Durch diese durch den Rechtsstaat vermittelten Eigentumsrechte wird die Verfügungsgewalt der Bürger über ihre Ressourcen geschützt und begrenzt. In Anlehnung an Acemoglu et al. (2005c, S. 397) wird im Folgenden der Begriff „ökonomische Institutionen“ als Synonym für Rechtsstaatlichkeit und die

\footnotetext{
${ }^{2}$ Der wohl bekannteste Demokratie-Indikator ist der „Freedom House Index“ oder „Gastil Index“, der die Qualität der demokratischen Strukturen auf einer Skala von 1 bis 7, wobei 7 das geringste Maß und 1 das höchste $\mathrm{Maß}$ an politischer Freiheit darstellt, quantifiziert. Relevante Charakteristika sind etwa die Existenz freier und fairer Wahlen, sowie wettbewerbsfähiger Parteien oder anderer politischen Gruppierungen, oder die Rolle von Wahlen für die Determinierung tatsächlicher politischer Macht. Ein anderer weit verbreiteter Index ist der Demokratie Index des Polity IV Projekts, der auf einer von 0 bis 10 reichenden Demokratie- und Autokratieskala basiert, die die meisten Staaten von 1800 bis zum Ende des 20. Jahrhunderts erfasst. Die für eine ,institutionalisierte Demokratie“ relevanten Faktoren sind der Umfang der politischen Beteiligung, die Rekrutierung der politischen Führung durch freie, offene Wahlen und die rechtliche Einschränkung der Exekutive (Muno, 2001).

${ }^{3}$ Beispiele für empirische Arbeiten mit dieser bivariaten Definition sind u.a. Persson und Tabellini (1994, 2004), Wacziarg (2001) und Tavares und Wacziarg (2001).

${ }^{4}$ Vergleiche dazu Hayek's Überblick über die Geschichte des Rechtsstaatsbegriffs, Hayek (2005, Kap. 11).
} 
Gewährung von Eigentumsrechten verwendet. Dabei ist anzumerken, dass vielen theoretischen Arbeiten ein weiter Demokratiebegriff, in dem effiziente ökonomische Institutionen gleichsam als Charakteristika von Demokratien behandelt werden, zugrunde liegt.

Die Frage nach der relevanten Definition von Wohlstand und wirtschaftlicher Entwicklung erlaubt ebenfalls unterschiedliche Interpretationen. Generell lassen sich zwei Sichtweisen unterscheiden. Zum einen beschreibt Wohlstand einen Zustand innerhalb eines gewissen politischen Systems, und wird z.B. durch das Pro-Kopf-Einkommen zu einem bestimmten Zeitpunkt gemessen. Zum anderen kann man Wohlstand und wirtschaftliche Entwicklung als dynamischen Prozess auffassen, reflektiert etwa durch die Geschwindigkeit des Wirtschaftswachstums während eines bestimmten Zeitraums. Während der Entwicklungsansatz von einem positiven Effekt des Entwicklungsstandes eines Landes ausgeht, untersucht die institutionelle Sichtweise zumeist die Auswirkungen demokratischer Strukturen auf das Wirtschaftswachstum. Dabei gibt es natürlich Ausnahmen, die gesondert besprochen werden.

Die Arbeit beantwortet die Grundfrage nach dem Zusammenhang zwischen Demokratie und Wohlstand, indem in Abschnitt 2 zuerst die empirischen Ergebnisse zur kausalen Wirkung von demokratischen Institutionen auf die wirtschaftliche Entwicklung beleuchtet werden. In Abschnitt 3 werden dann die Ergebnisse zum Entwicklungsansatz, demzufolge wirtschaftliche Entwicklung die Demokratisierung beeinflusst, diskutiert. Die meisten diskutierten Arbeiten untersuchen jedoch nicht oder nicht nur direkte Effekte, sondern vielmehr indirekte Effekte, die über mehrere Wirkungskanäle laufen können. In Anbetracht der unterschiedlichen Implikationen und der praktischen Relevanz wird in beiden Abschnitten die Literatur daher anhand der unterschiedlichen Wirkungskanäle gegliedert, wobei zu jedem Kanal die wichtigsten jüngeren Arbeiten besprochen werden. In Anlehnung an Lipsets (1959) Bedingungen für eine funktionierende Demokratie werden die aus ökonomischer Sicht relevantesten indirekten Wirkungskanäle in die Kategorien „ökonomisch“, „polit-ökonomisch“ und „,institutionell“ eingeteilt. ${ }^{5}$ Dabei stellen ökonomische Kanäle einen Zusammenhang zwischen Demokratie und Wohlstand über Mechanismen wie die Akkumulation von Ressourcen her. Polit-ökonomische Kanäle konzentrieren sich auf Zusammenhänge, die auf die mit der Ungleichheit der Lebensbedingungen verbundene Umverteilung, oder auf durch Interessenskonflikte getriebene Politikmaßnahmen zurückgehen. Institutionelle Wirkungskanäle zwischen Demokratie und Wohlstand spiegeln den Einfluss ökonomischer Institutionen im Sinne von Rechtsstaatlichkeit

\footnotetext{
${ }^{5}$ Lipset's (1959) “economic development index “ enthält die folgenden Indikatoren, die aus modernisierungstheoretischer Sicht als begünstigende Bedingungen für eine Demokratie in Frage kommen: ein relativ hohes Niveau sozioökonomischer Entwicklung in einer kapitalistischen Marktökonomie; ein hoher Bildungsstand; eine große und wachsende Mittelklasse sowie eine Unterschicht, die auf ein hohes Maß sozialer und wirtschaftlicher Sicherheit zählen kann; eine relativ offene Klassenstruktur; ein relativ egalitäres Wertesystem; und eine hohe Beteiligung an freiwilligen Organisationen.
} 
und Eigentumsrechten wider. Diese Kategorisierung soll lediglich der Strukturierung der Beiträge dienen, und erhebt keinen Anspruch auf Allgemeingültigkeit. Tatsächlich stellt sich beispielsweise ein Wachstumseffekt durch ökonomische Institutionen letztlich mittelbar über verbesserte Bedingungen zur Bildung von (Human-)Kapital ein. Um die unterschiedlichen Zusammenhänge nicht zu verwischen, vor allem aber um einen Kernpunkt der aktuellen Debatte herauszuarbeiten, wird der Unterscheidung zwischen ökonomischen Institutionen, der „Rechtsstaatlichkeit“, und politischen Institutionen, der „Demokratie“, besondere Aufmerksamkeit gewidmet. Die Arbeit endet mit einer kurzen Zusammenfassung und einer Diskussion über Implikationen für die Entwicklungspolitik.

\section{Von der Demokratie zu wirtschaftlicher Entwicklung und Wirtschaftswachstum}

\subsection{Hintergrund und Evidenz für direkte Effekte}

Der institutionelle Ansatz, nach dem die Implementierung und Existenz von politischen (demokratischen) Strukturen die wirtschaftliche Entwicklung bestimmt, hat vor allem im letzten Jahrzehnt großes Interesse in der Forschung gefunden. Entgegen der Sicht der Modernisierungstheorie, die einen positiven Zusammenhang zwischen Entwicklungsstand und Demokratie vertritt und im nächsten Abschnitt diskutiert wird, sind sich die Vertreter des institutionellen Ansatzes nicht einig, ob Demokratie gut oder hinderlich für die dynamische wirtschaftliche Entwicklung, d.h. für das Wirtschaftswachstum, ist.

Kurzman et al. (2002) sehen die Anfänge der Diskussion bereits im 17. Jahrhundert, geführt von Thomas Hobbes, der als Pionier der „trade-off“ Sicht gilt. In seinem Werk „The Leviathan“ (1651) argumentiert er, dass absolutistische Regime eher gewillt sind, das Allgemeinwohl zu verbessern, allein schon deshalb, weil sie sonst ihre eigenen Interessen nicht vertreten könnten. ${ }^{6}$ Regime mit eingeschränkter Macht hingegen teilen sich in Interessensgruppen, die bloß vom Unglück des Volkes profitieren wollen. In „The Commonwealth of Oceania“ (1656) wird dem von James Harrington Hobbes widersprochen. Er ist der Überzeugung, Herrscher seien potentielle Plünderer deren Macht dementsprechend eingeschränkt werden sollte. Damit steht Harrington am Anfang der „win-win“ Perspektive, die die Behauptung Lipsets umgekehrt und argumentiert, dass erst ein demokratischer Staat wirtschaftliche Entwicklung vorantreibt. ${ }^{7}$ Das

\footnotetext{
${ }^{6}$ Eine radikalere Sicht vertritt in diesem Zusammenhang Gregor (1979; in Kurzman et al, 2002), der die Demokratie als einen ineffizienten Luxus der reichen Länder kritisiert und für eine „Entwicklungsdiktatur“ vor allem in den ärmeren Ländern plädiert, in der das Volk eine Art protestantische Arbeitsethik aufgezwungen bekommen sollte.

${ }^{7}$ So argumentiert etwa Sklar (1987, in Kurzman et al, 2002), Wachstum brauche eine „Entwicklungsdemokratie“, in der willkürliche Machtausübung durch Gesetze und Wahlen eingegrenzt wird und dem Volk somit die Sicherheit gegeben wird, für ihre wirtschaftliche Zukunft zu planen
} 
Hauptargument dieser Perspektive ist, dass eine Diktatur die Rechtsstaatlichkeit, die für wirtschaftliche Aktivität essenziell ist, untergraben würde.

Diese konträren Standpunkte bestimmen noch immer die Diskussion. Die Argumente zugunsten der ,win-win“ Perspektive erhalten in jüngster Zeit zunehmend empirische Unterstützung. Vor allem neuere Studien, die Identifikation nicht über Querschnittsvariation, sondern über zeitliche Variation innerhalb eines Landes herstellen, finden einen positiven kausalen Zusammenhang zwischen Regimeveränderungen bzw. demokratischen Reformen und wirtschaftlicher Entwicklung. Beispiele hierfür sind die Arbeiten von Papaioannou und Siourounis (2004), Pettersson (2004) und Rodrik und Wacziarg (2005). ${ }^{8}$

Allerdings sind die Ergebnisse zur Rolle von Demokratie für Wachstumseffekte Gegenstand einer Debatte. Zum einen scheint der unmittelbare Effekt demokratischer Reformen nicht sehr groß zu sein, Demokratien brauchen demnach Zeit, um ihre dem wirtschaftlichen Wohlstand förderlichen Wirkungen zu entfalten. Zum anderen gibt es auch Kritiker, die einen direkten kausalen Zusammenhang bezweifeln und empirische Effekte auf die Vernachlässigung der tatsächlich treibenden Mechanismen zurückführen. Vertreter dieser Sichtweise argumentieren, Wachstum sei letztlich durch die Akkumulation von Faktoren wie physischem Kapital oder Humankapital getrieben, die wiederum nur funktionierenden ökonomischen Institutionen, also Eigentumsrechten und Rechtsstaatlichkeit, geschuldet sei. Demokratie selbst nimmt in dieser Argumentation eine untergeordnete, und höchstens instrumentelle Rolle ein. Vertreter dieser Argumentation sind unter anderem Barro (2000) und Glaeser et al. (2004). Gestützt wird diese differenzierte Sichtweise von empirischen Ergebnissen wie beispielsweise der Studie von Tavares und Wacziarg (2001), die unter Verwendung von Panel-Daten für 65 Länder für den Zeitraum 1970 bis 1989 ein System schätzen bestehend aus einer Gleichung für das Wirtschaftswachstum, die von acht unterschiedlichen strukturellen Wirkungskanälen beeinflusst wird. Demokratie selbst wird als endogen aufgefasst und geeignet durch exogene Variablen instrumentiert. Im Ergebnis finden Tavares und Wacziarg einen moderat negativen Gesamteffekt von Demokratie auf das Wirtschaftswachstum, was primär für die trade-off Sicht spricht. Allerdings zeigen die Resultate für die einzelnen strukturellen Wirkungskanäle, wie dieser negative Zusammenhang zustande kommt. In der Tat veranschaulichen die Ergebnisse von Tavares und Wacziarg, dass sich demokratische Strukturen positiv auf die Bildung von Humankapital, jedoch negativ auf die Bildung von physischem Kapital auswirken, und somit durch ökonomische Kanäle die wirtschaftliche Entwicklung beeinflussen. Weitere negative Wachstumseffekte treten infolge von Ungleichheit sowie größerem Staatskonsum und höherer Abgabenlast zutage.

\footnotetext{
${ }^{8}$ Politische Variablen haben auch einen signifikanten Effekt in Sala-i-Martin's (1997) Studie über die empirische Relevanz aller gängigen Determinanten in Wachstumsregressionen.
} 
Diese Diskussion, die im oberen Teil von Tabelle 1 zusammengefasst ist, macht deutlich, dass die Frage nach einem (direkten) Effekt von demokratischen Strukturen auf die wirtschaftliche Entwicklung eines Landes noch nicht abschließend beantwortet ist. Im Licht der jüngsten empirischen Ergebnisse scheint jedoch die „win-win“ Perspektive, die von einem positiven, möglicherweise aber indirekten Zusammenhang ausgeht, empirisch plausibel (für eine Übersicht siehe Tabelle 1). Diese Diskussion verdeutlicht jedoch auch die Notwendigkeit, die empirische Relevanz möglicher indirekter Wirkungskanäle genauer zu untersuchen.

\section{2 Ökonomische Kanäle}

Akkumulation von Kapital. Nach herrschender Meinung sind die Akkumulation von physischem Kapital und Humankapital die treibenden Kräfte und die entscheidenden Determinanten des Wirtschaftswachstums. ${ }^{9}$ Ein, wenn auch indirekter, kausaler Demokratieeffekt impliziert, dass demokratische Strukturen den Aufbau dieser Ressourcen über die Zeit hinweg begünstigen und beschleunigen. Darüber, wie Demokratie auf Kapitalakkumulation einwirken kann, existieren allerdings unterschiedliche Auffassungen. Tavares und Wacziarg (2001) nennen verschiedene Möglichkeiten. Der politische Prozess kann einerseits $\mathrm{zu}$ einer Umverteilung des Staatseinkommens zugunsten von Arbeit und zum Nachteil für Kapital führen, weil Gewerkschaften und Arbeitnehmerinteressen in Demokratien mehr Einfluss haben. Die in der Folge höhere Verhandlungsmacht im Verteilungskampf um Renten führt zu höheren Löhnen und niedrigeren Kapitalerträgen und senkt somit die Anreize, private Investitionen zu unternehmen. Andererseits können demokratische Strukturen höhere Kapitalerträge mit sich bringen, insbesondere wenn dadurch Eigentumsrechte transparenter und besser geschützt sind und somit wirtschaftliches Handeln begünstigt wird. Den gleichen Effekt erzielen demokratische Strukturen, falls sie den Grad an politischer, sozialer und wirtschaftlicher Unsicherheit verringern. Auf die Rolle der Ausgestaltung von Eigentumsrechten, also etwa die entscheidenden Rahmenbedingungen für die Kapitalversorgung, wird in Abschnitt 2.4 gesondert eingegangen. Direkte Evidenz scheint jedoch für einen negativen Effekt von Demokratie zu sprechen. Tavares und Wacziarg finden in ihren Regressionen Evidenz für einen negativen Einfluss von Demokratien auf die Akkumulation physischen Kapitals, die ihrerseits das Wachstum beschleunigt. Konsistent damit zeigen die Ergebnisse von Minier (1998), dass Kapitalakkumulation vor allem in Ländern mit einem relativ hohen Bildungsniveau aber mit weniger demokratischen Strukturen, einen starken Effekt auf Wachstum hat.

Humankapitalakkumulation. Die gängige Argumentation hinsichtlich des Humankapital-Kanals zwischen Demokratie und Wirtschaftswachstum besagt, dass Demokratien die

\footnotetext{
${ }^{9}$ Kapitalakkumulation ist die treibende Kraft im neoklassischen Wachstumsmodell, siehe Solow (1956), während Lucas (1988) die Rolle des Humankapitals für die Wachstumstheorie betonte.
} 
Humankapitalakkumulation besonders fördern, da der Umfang an öffentlichen Dienstleistungen, insbesondere auch im Bildungssektor, größer ist als in nicht-demokratischen Staaten. Eine Bevölkerung, die gute Ausbildungsmöglichkeiten und zukünftige Weiterbildungs- und Förderungsprogramme zur Verfügung gestellt bekommt, bildet aber die Grundlage für wirtschaftliche Entwicklungsfähigkeit. Vor dem Hintergrund der historisch heterogenen Entwicklung in Amerika argumentieren Galor, Moav und Vollrath (2005), dass geographische Bedingungen, die sich in der Verteilung von Grundbesitz widerspiegeln, in manchen Regionen zur Entstehung einer Klasse von wohlhabenden Grundherren geführt haben. Diese Elite hat, so das Argument, die Einführung von Humankapital fördernden Institutionen nachteilig beeinflusst, was zu divergierenden Wachstumspfaden in verschiedenen Regionen und Ländern der Welt geführt haben könnte.

Lott (1999) untersucht den Zusammenhang zwischen den Ausgaben für staatliche Ausbildung und dem Auftreten totalitärer Regimes, insbesondere die Frage, ob totalitäre Staaten mehr für Ausbildung ausgeben als demokratische. Lotts Modell zufolge sollte ein höherer Grad an Totalitarismus sowie ein höheres pro Kopf Einkommen die Ausbildungsausgaben tendenziell erhöhen, die Wichtigkeit von Indoktrination in reicheren totalitären Regimes jedoch zurückgehen. In Regressionen mit Querschnittsdaten aus 99 Ländern findet Lott seine erste Hypothese bestätigt, während die Evidenz für die Hypothese, die Ausgaben für Indoktrination seien in reicheren Staaten geringer, nicht eindeutig ist. Den Umstand, dass jedoch Bildungsausgaben, insbesondere wenn sie der Indoktrination dienen, nicht gleich bedeutend sind mit effektiver Humankapitalbildung, zeigen jedoch neuere Ergebnisse, die einen direkten Bezug zum Wachstumspotenzial herstellen. Unter Verwendung von Paneldaten finden sowohl Minier (1998) als auch Tavares und Wacziarg (2001) und Wacziarg (2001) starke empirische Evidenz dafür, dass Demokratien bessere Bedingungen für die Akkumulation von Humankapital aufweisen, wodurch ein positiver Wachstumsimpuls angeregt wird. Minier stößt zudem auf einen negativen Effekt von Humankapital in weniger demokratischen Ländern, den sie dadurch erklärt, dass demokratische Länder ihren Bürgern mehr Auswahl- und Betätigungsmöglichkeiten bieten, was letztlich zu höheren Ausbildungsrenditen führt.

Dass Humankapital, und nicht politische Institutionen wie Demokratie, der Hauptmotor des Wirtschaftswachstums ist, wird aus einer ähnlich gelagerten Untersuchung von Glaeser et al. (2004) deutlich, die mit Hilfe von Paneldaten die relative empirische Relevanz von Institutionen und Humankapital für die wirtschaftliche Entwicklung erforschen. Gute Politik und ökonomische Institutionen, die beispielsweise die Eigentumsrechte aller Bürger garantieren, sind gemäß der Ergebnisse dieses Beitrags zwar wichtiger als die pure Existenz demokratischer Strukturen, aber weniger wichtig als Humankapitalbildung. Die Ergebnisse dieser Studie legen 
umgekehrt nahe, dass demokratische Strukturen das Ergebnis von Armutsbekämpfung und erfolgreicher wirtschaftlicher Entwicklung sein können. Zusammenfassend bleibt festzuhalten, dass demokratische Länder zwar möglicherweise bessere Bedingungen für die Humankapitalakkumulation, den vermutlich wichtigsten Wachstumsfaktor, bieten. Aber auch hier bestehen Zweifel bezüglich der essenziellen Rolle demokratischer Strukturen für durch Humankapital getriebenes Wachstum.

\subsection{Polit-ökonomische Kanäle}

Ungleichheit, Umverteilung und die Größe des Staates. Die Meinungen zum Effekt von Einkommensungleichheit als Kanal zwischen Demokratie und Entwicklung sind geteilt. Je größer die wirtschaftliche Ungleichheit zwischen arm und reich, desto größer das Verlangen derjenigen am unteren Ende der Einkommens- und Wohlstandsskala nach Umverteilung. Im einfachsten Modell einer Demokratie determiniert der Medianwähler, dessen Einkommen unter dem des Durchschnittseinkommens liegt, die Größe des Umverteilungshaushaltes und somit die Staatsquote. Größere Ungleichheit hat demnach größere Umverteilung zur Folge, siehe Meltzer und Richard (1981). Wenn aber mit höherer Umverteilung größere allokative Ineffizienzen, etwa durch steuerbedingte Verzerrungen der Arbeitsanreize, einhergehen, so sind geringerer Wohlstand und langsameres Wachstum die Folge. Demokratische Reformen, die eine Erweiterung der politischen Mitwirkung von niedrigeren Einkommensschichten implizieren, würden demnach über höhere direkte progressive Umverteilung über Steuern und Subventionen, oder Umverteilung über die Bereitstellung von Wohlfahrtsprogrammen $\mathrm{zu}$ höheren Wohlfahrtsverlusten führen. Wird Politik in Demokratien stärker durch Interessensgruppen beeinflusst, deren Bedürfnisse durch staatliche Interventionen befriedigt werden, so führt das, dem Argument von Olson (1982) folgend, zu zusätzlichen Verzerrungen. Ähnliches gilt für die von Niskanen (1971) beschriebene Tendenz, dass einmal geschaffene Bürokratien sich mit der Zeit verselbständigen und erweitern um das Ausmaß ihres Einflusses zu vergrößern. Andererseits schafft mehr Umverteilung in Demokratien jedoch möglicherweise ein egalitäreres und friedlicheres, und damit investitionsfreundlicheres, gesellschaftliches Klima und infolge dessen langfristig Wachstum.

Persson und Tabellini (1994) schätzen Wachstumsgleichungen, die Ungleichheit und politische Strukturen getrennt als erklärende Variablen aufnehmen. Ihre Resultate legen nahe, dass Volkswirtschaften mit größerer Gleichheit der Bevölkerung schneller wachsen, jedoch nur wenn sie demokratisch sind. Perotti (1996) beleuchtet in seiner Analyse den Effekt verzerrender Besteuerung. Er argumentiert, dass sinkende Einkommensungleichheit zu weniger umverteilender Besteuerung führt, was wiederum das Wirtschaftswachstum anregt. In seinen Regressionen findet er jedoch keine robuste Evidenz dafür, dass der Zusammenhang zwischen 
Ungleichheit, Besteuerung und Wachstum in Demokratien stärker ist als in nicht-demokratischen Ländern. Tavares und Wacziarg (2001) untersuchen, inwieweit Demokratie zu weniger Einkommensungleichheit und letztlich höherem Wachstum führt. In der Tat finden sie einen negativen Effekt von Demokratie auf Ungleichheit und einen kleinen positiven Effekt von Einkommensegalität auf Wirtschaftswachstum. In ähnlicher Weise untersuchen Tavares und Wacziarg (2001) die Rolle von demokratischen Strukturen für die Größe des Staatsapparates und seine Struktur hinsichtlich Besteuerung und Verzerrungen. Den Schätzungen zufolge weisen Demokratien tatsächlich tendenziell größere Staatsapparate auf, die sich negativ auf die Wachstumsrate auswirken. Die Ergebnisse zeigen ebenfalls einen negativen Wachstumseffekt der Größe der Verzerrungen, die jedoch in Demokratien nicht größer zu sein scheinen als in nicht-demokratischen Systemen. Papaioannou und Siourounis (2004) stützen diese Ergebnisse, und finden ebenfalls einen negativen Wachstumseffekt von Demokratie über die Staatsgröße. Mulligan et al. (2004) erhalten anhand von Querschnittsdaten keine Anzeichen für signifikante Unterschiede zwischen Demokratien und Nicht-Demokratien hinsichtlich Staatskonsum, Umverteilung, oder Bildungsausgaben.

Handelsliberalisierung und Natürliche Ressourcen. Einen weiteren möglichen Kanal zwischen Demokratie und Wachstum stellen Politikmaßnahmen wie die Einbindung in internationale Handelsbeziehungen bzw. Offenheit für den Handel dar. Der Grad an Handelsfreiheit wird unter anderem durch den Grad an politischer Freiheit bestimmt. Einerseits sind Schutzzölle, die meistens im Sinne von Interessensgruppen aber zum Nachteil der gesamtwirtschaftlichen Effizienz sind, in Demokratien schwieriger zu implementieren. Andererseits kann es in Anbetracht von Lobbyismusaktivitäten, die sowohl in Autokratien, wie auch in Demokratien an der Tagesordnung stehen, sein, dass die Gegner des Freihandels auch in Demokratien leichter zu mobilisieren sind als dessen Anhänger. Eine besondere Rolle kommt dabei der Ausstattung mit natürlichen Ressourcen zu. Deren Ausbeutung durch Staatsorgane oder private Unternehmen begünstigt zwar die Offenheit, hemmt aber möglicherweise damit langfristiges Wachstum.

In einer frühen Studie zu den Wachstumseffekten von Handelspolitik und Handelsoffenheit argumentieren Sachs und Warner (1995) auf Basis von Ergebnissen, die mit Querschnittsdaten gewonnen wurden, dass handelsfreundliche Politik eine hinreichende, wenn auch nicht notwendige Bedingung für Wirtschaftswachstum ist. Tavares und Wacziarg (2001) können diese Ergebnisse nicht bestätigen. Sie finden zwar einen positiven Zusammenhang zwischen Demokratie und Handelsoffenheit, aber keine Evidenz für einen Wachstumseffekt. Die Ergebnisse von Papaioannou und Siourounis (2004) sind positiver hinsichtlich eines solchen Wachstumseffekts. Ein Überblick über die Wachstumseffekte von Politikmaßnahmen ergibt jedoch, dass diese Effekte generell nicht sehr robust erscheinen, insbesondere im Hinblick auf 
die Inklusion von politischen und ökonomischen Institutionen, siehe die Diskussion in Easterly (2005).

Instabilität. Die Stabilität der Staatsführung ist eine wichtige Eigenschaft politischer Systeme. Politische Instabilität führt zu Unsicherheit über die zukünftige Politik und verzerrt so Investitionsanreize. Zugleich erzeugt politische Unsicherheit Anreize für herrschende Eliten, sich die Ressourcen der Wirtschaft anzueignen solange dies möglich ist. Für den Fall, dass demokratische Strukturen politische Instabilität reduzieren, schaffen sie weniger Unsicherheiten als autokratische Staaten und fördern somit Investitionen und Wachstum. Weder Perotti (1996) noch Tavares und Wacziarg (2001) finden jedoch in ihren empirischen Untersuchungen statistisch signifikante Evidenz für einen positiven Wachstumseffekt von Demokratie über politische Stabilität. Die Ergebnisse von Treisman (2000) deuten jedoch darauf hin, dass die mit Demokratien verbundene politische Stabilität langfristig positive indirekte Wachstumseffekte entfalten kann, beispielsweise indem Korruption reduziert wird. Anstelle des Einflußes von Demokratie auf politische Stabilität untersucht Mobarak (2005) den Zusammenhang zwischen Demokratie und wirtschaftlicher Stabilität. Seine Ergebnisse zeigen, dass Demokratie die Volatilität des Wirtschaftswachstums dämpft. Niedrigere Volatilität und höhere wirtschaftliche Stabilität führt jedoch wiederum zu höheren durchschnittlichen Wachstumsraten. Außer diesem indirekten Volatilitätseffekt findet er keinen direkten Effekt von Demokratie auf Wachstum.

Zusammenfassend lassen die empirischen Ergebnisse $\mathrm{zu}$ polit-ökonomischen Einflussfaktoren kein eindeutiges abschließendes Urteil über die Vorteilhaftigkeit von demokratischen Strukturen zu, siehe auch die Aufstellung in Tabelle 1. Die Evidenz spricht jedoch eher für einen positiven Demokratieeffekt durch polit-ökonomische Kanäle, hauptsächlich durch die mit Demokratien verbundene niedrigere wirtschaftliche Ungleichheit.

\section{4 Ökonomische Institutionen: Rechtsstaatlichkeit}

Es herrscht in der Literatur Einigkeit darüber, dass Institutionen insgesamt eine wichtige Rolle für die langfristige wirtschaftliche Entwicklung spielen. Umstritten ist jedoch, welche Institutionen ausschlaggebend sind. Während Demokratie im Wortsinne nur den Prozess der Rechtsdeterminierung festlegt, wird in der Literatur Demokratie oft mit der Isonomie, der Gleichheit aller Menschen vor dem Gesetz, gleichgesetzt. Oft nehmen theoretische Arbeiten vor diesem Hintergrund der Einfachheit halber an, dass demokratische Strukturen unter Effizienzgesichtspunkten überlegen sind, etwas weil sie besseren Schutz des Eigentums gewähren. ${ }^{10}$ Dagegen gibt es eine Strömung in der Literatur, die in der Rechtsstaatlichkeit bzw. der Garantie von Eigentumsrechten wichtigere Entwicklungsfaktoren sieht als in

\footnotetext{
${ }^{10}$ Beispiele hierfür sind etwa die Artikel von Olson (1993) oder Gradstein (2006).
} 
demokratischen Strukturen. Hayeks Standpunkt „Demokratie ist Mittel, nicht Zweck“ spiegelt diese Sichtweise wider (Hayek, 2005, S. 135).

Diese Rechte können sowohl durch formelle Institutionen (d.h. als Teil der Verfassung und ihre Organe) wie auch durch informelle Institutionen im Sinne von North (1990) (bspw. als Teil von Verhaltensnormen) sichergestellt werden. Werden Eigentumsrechte effektiv durchgesetzt und von jedermann respektiert, so werden durch sie letztlich auch die Kosten bestimmt, die für einen Verstoß anfallen. Die Implementierung von Eigentumsrechten unterliegt Institutionen wie insbesondere rechtlichen Normen, sowie einer funktionierenden und neutralen Judikative und Exekutive. Eigentumsrechte wiederum geben den Menschen Anreize, Güter zu erzeugen, zu investieren und Innovationen hervorzubringen, da durch sie die Erträge vor Ausbeutung geschützt sind.

Empirisch ist das Ausmaß und die Qualität von Eigentumsrechten nur schwer zu messen. Die frühe empirische Literatur benutzte aus Mangel an besseren Daten Maße politischer Stabilität, wie z.B. die Häufigkeit von Putschen, Revolutionen und politischen Attentaten als Indikator. Daneben fanden auch Demokratieindizes breite Verwendung (siehe etwa Barro, 1991). Erst neuere Studien, beginnend mit Knack und Keefer (1995), Hall und Jones (1999) sowie Rodrik et al. (2004) benutzen Variablen, die dem Konzept von Eigentumsrechten näher sind und verwenden Indikatoren von privaten internationalen Anlagerisikodiensten, wie der ,International Country Risk Guide“ (ICRG) und der „Business Environment Risk Intelligence“ (BERI). Elemente wie Vertragsvollstreckbarkeit und Enteignungsrisiko sind Hauptbestandteil dieser Indikatoren.

Knack und Keefer (1995) finden unter Verwendung direkter Indikatoren von Eigentumsrechten größere Effekte auf Investitionen und Wachstum als unter Verwendung von Gastils Index oder anderer Variablen politischer Stabilität. ${ }^{11} \mathrm{Wu}$ und Davis (1999) weisen darauf hin, dass die Wirtschaftswachstumsrate unabhängig von politischer Freiheit und dem Einkommensniveau ist, sobald man die ökonomische Freiheit in die Regression aufnimmt. Umgekehrt ist, gegeben das Einkommensniveau, politische Freiheit unabhängig von ökonomischer Freiheit und der Wachstumsrate. Demnach scheinen ökonomische Freiheiten eine wichtige Vorraussetzung für Wachstum. Ein hohes Einkommensniveau scheint dagegen eine Bedingung für einen hohen Grad an politischer Freiheit zu sein. Wu und Davis bestätigen damit indirekt Lipsets Modernisierungsansatz. Barro (2000) beleuchtet die Wechselwirkungen zwischen Demokratie und Rechtsstaatlichkeit. Seine Ergebnisse führen ihn zu dem Schluss, dass

\footnotetext{
${ }^{11}$ Hall und Jones (1999) untersuchen die Determinanten des Pro-Kopf Einkommensniveaus. Ihre Resultate betonen die positive Rolle von „,sozialer Infrastruktur“, im Sinne von Eigentumsrechten und Rechtstaatlichkeit, sowie Handelsoffenheit. Dollar und Kraay (2003) finden einen positiven langfristigen Effekt von Rechtsstaatlichkeit auf das das Wohlstandsniveau, aber einen negativen Effekt von politischer Freiheit. Heitger (2003) kommt zu ähnlichen Ergebnissen.
} 
Demokratie nicht unbedingt notwendig für Wachstum ist - ebensowenig wie Diktatur. Für ein Land mit schwachen Institutionen (d.h. ein geringes Maß an Demokratie und Rechtsstaatlichkeit) sind nach seinen Ergebnissen demokratische Reformen weniger wichtig als eine Erweiterung der Rechtsstaatlichkeit, um Wachstum und Investitionen zu fördern. Darüber hinaus findet Barro keine Evidenz für eine direkte Rolle von Demokratie in der Implementierung von Rechtsstaatlichkeit. Selbst wenn Demokratie das Endziel sein soll, sollten sich Barro zufolge arme Länder eher auf Rechtsstaatlichkeit, Eigentumsrechte und freie Märkte konzentrieren als sich an die Implementierung von Demokratie festzuklammern. Auch Acemoglu, et al. (2005c) sehen die Unterschiede in ökonomischen Institutionen als die fundamentale Ursache für Unterschiede in wirtschaftlicher Entwicklung. Das wirtschaftliche Potential hängt ihren Untersuchungen zufolge insbesondere davon ab, wie eine Wirtschaft organisiert ist, sprich, von ihren ökonomischen Institutionen. Die Ursache für unterschiedliche ökonomische Institutionen liegt demnach in der Verteilung und Ausgestaltung von Macht sowie der Beschaffenheit von politischen Institutionen. Wachstumsfördernde ökonomische Institutionen entstehen dann, wenn politische Institutionen die Macht an die Gruppen verteilen, die Interesse an der Durchsetzung von Eigentumsrechten haben, wenn sie Machtinhaber effektiv einschränken und wenn die Machtinhaber geringe Renten abschöpfen können. Als weiteres Attribut einer funktionierenden Demokratie kann man auch die Gewaltenteilung auffassen. In diesem Zusammenhang lassen sich die Ergebnisse von Feld und Voigt (2003), dass Unabhängigkeit der Justiz ein wachstumsförderndes Element darstellt, als Evidenz für diesen Wirkungskanal interpretieren. ${ }^{12}$ Auch Rigobon und Rodrik (2004) finden in ihren Analysen eine Wechselwirkung zwischen ökonomischen und politischen Institutionen. Dagegen stehen Glaeser et al. (2004) den allgemein verwendeten Indikatoren zur Messung ökonomischer Institutionen skeptisch gegenüber. Rodrik et al. (2004) weisen ebenfalls auf die Unzulänglichkeit ihres Indikators hin, da er allein von den subjektiven Einschätzungen von Investoren und anderen Beobachtern abhängig ist. Dennoch finden sie heraus, dass ökonomische Institutionen wichtiger im Entwicklungsprozess sind als Geographie und Integration. Ihrer Ansicht nach sind diese Indikatoren nicht geeignet, eine kausale Verbindung zwischen ökonomischen Institutionen und Wachstum zu diagnostizieren, und sie finden in der Folge auch keine empirische Evidenz dafür, dass institutionelle Faktoren das Wachstum treiben. Im Vergleich zur Bildung von Humankapital und Sozialkapital, welches die institutionellen und produktiven Kapazitäten einer Gesellschaft formt, haben Institutionen ihren Ergebnissen zufolge nur einen zweitrangigen Effekt auf wirtschaftliche Entwicklung

Neben fehlender Kontrolle gegen Ausbeutung durch den Staat ist auch das Auftreten von Korruption ein Zeichen für mangelnde Rechtsstaatlichkeit. Korruption wirkt sich empirisch

\footnotetext{
${ }^{12}$ Feld und Voigt (2003) unterscheiden zwischen formeller (de jure) und tatsächlicher Unabhängigkeit der Judikative, wobei vor allem letzterer Entscheidende Bedeutung für die wirtschaftliche Entwicklung zukommt.
} 
negativ auf private Investitionen aus und ist damit wachstumsfeindlich. ${ }^{13}$ Mehrere jüngere Beiträge zeigen, dass Korruption in Demokratien generell weniger ausgeprägt ist. Während Ades und Di Tella (1999) mit Querschnittsdaten nur schwach positive Effekte von politischen Rechten auf Korruption finden, zeigt Treisman (2000) mit Querschnittsdaten aus mehreren Jahren, dass Demokratie an sich zwar keinen direkten Effekt auf das Auftreten von Korruption zu besitzen scheint, dass aber die Verwaltung von Ländern, die über längere Zeit demokratische Strukturen besitzen, weniger korrupt ist. ${ }^{14}$ Chowdhury (2004) und Lederman et al. (2005) schließlich finden mit Hilfe von Längsschnitts- und Paneldaten über mehrere Länder Evidenz dafür, dass Demokratie und damit verbundene Charakteristika wie Pressefreiheit im Speziellen, und politische Stabilität im Allgemeinen, das Auftreten von Korruption negativ beeinflussen. Soweit Korruption die wirtschaftliche Entwicklung hemmt, ist dies zumindest indirekte Evidenz für die institutionelle Sicht.

Insgesamt wird aus den empirischen Untersuchungen $\mathrm{zu}$ den Wachstumseffekten von Demokratie, illustriert in Tabelle 1, deutlich, dass Wachstumsimpulse hauptsächlich von verbesserten Bedingungen hinsichtlich Ausbildung, Ungleichheit und vor allem ökonomischen Institutionen ausgehen.

\section{Von wirtschaftlicher Entwicklung zur Demokratie}

\subsection{Hintergrund und Evidenz für direkte Effekte}

Spätestens seit Veröffentlichung von Lipsets Artikel in 1959 steht die Vermutung im Raum, dass wohlhabende Staaten mit höherer Wahrscheinlichkeit Demokratien implementieren und aufrechterhalten als arme Staaten. Diese Idee wird häufig die Aristoteles-Lipset-Hypothese genannt. ${ }^{15}$ Seit den 1990er Jahren haben mehrere Forscher Lipsets Theorie aufgegriffen und versucht, ihre empirische Gültigkeit zu überprüfen. ${ }^{16}$ Dabei war bis vor kurzem herrschende Meinung, dass ein positiver, direkt kausaler Effekt vom Entwicklungsstand einer Volkswirtschaft auf die Implementierung einer demokratischen Staatsform existiert (siehe Tabelle 2 für eine Übersicht entsprechender Arbeiten). Glaeser et al. (2004) interpretieren ihre Evidenz jedoch dahingehend, dass Armutsreduktion und wirtschaftliche Entwicklung die Implementierung

\footnotetext{
${ }^{13}$ Siehe Mauro (1995) für eine frühe Studie mit Querschnittsdaten für 58 Länder.

${ }^{14}$ Ein ähnlicher Eindruck drängt sich bei einem internationalen Vergleich des Ausmaßes der Schattenwirtschaft auf, die in OECD Ländern weit weniger verbreitet scheint als etwa in afrikanischen Ländern, siehe Schneider (2004).

${ }^{15}$ Lipset führt den Grundgedanken auf Aristoteles zurück: "Seit Aristoteles ist immer wieder argumentiert worden, dass die Masse der Bevölkerung nur in einer wohlhabenden Gesellschaft, in der verhältnismäßig wenige Bürger in wirklicher Armut leben, wahrhaft am politischen Leben teilnehmen und genügend Selbstbeherrschung aufbringen können, um den Verlockungen verantwortungsloser Demagogen zu widerstehen“ (Lipset, 1960, eigene Übersetzung). Dabei bezieht sich Lipset, im Gegensatz zur vorliegenden Studie in erster Linie auf die Stabilität demokratischer Strukturen.

${ }^{16}$ Beispiele sind Huntington (1991), Burkhart und Lewis-Beck (1994), Przeworski und Limongi (1997), Barro (1999), Wu und Davis (1999), Boix und Stokes (2003), Acemoglu und Robinson (2003), Acemoglu et al. (2005b) und Glaeser et al. (2004).
} 
ökonomischer Institutionen, wie der Sicherstellung von Eigentumsrechten, vereinfachen oder erst ermöglichen könnte. In der Folge könnte sich dies auch positiv auf die Qualität politischer Institutionen auswirken. Acemoglu et al. (2005b) hingegen nutzen Längsschnittsdaten, um den kausalen Effekt des Entwicklungsstandes im Sinne von pro-Kopf Einkommen auf die Wahrscheinlichkeit demokratischer Strukturen zu schätzen. Ihre Resultate widerlegen frühere Ergebnisse aus Querschnittsdaten, die auf kausale Effekte des wirtschaftlichen Entwicklungsstandes schließen ließen. Diese Evidenz, so die Schlussfolgerung, ist hauptsächlich durch unbeobachtbare Heterogenität und Variation zwischen Ländern - also historische Faktoren - getrieben, und daher inkonsistent. Sobald Variation innerhalb der Länder zur Identifikation herangezogen wird, lassen sich keine direkten kausalen Effekte mehr belegen. Vor diesem Hintergrund gewinnt die Untersuchung möglicher indirekter Wirkungskanäle besonders an Bedeutung.

\section{2 Ökonomische Kanäle}

Humankapital und Bildungsstand. Die ökonomische Theorie und eine umfangreiche empirische Literatur suggerieren, dass der wirtschaftliche Entwicklungsstand einer Volkswirtschaft eng mit dem Ausbildungsstand der Bevölkerung assoziiert ist. Reichere Länder haben eine Bevölkerung mit höherem Bildungsstand, der wiederum wichtig für die Entstehung von demokratischen Institutionen ist. ${ }^{17}$

Barro (1999) testet diese These und findet, dass ein höherer Bevölkerungsanteil mit absolvierter Grundschulausbildung sowie ein geringerer Unterschied zwischen Männern und Frauen hinsichtlich Grundschulausbildung die Wahrscheinlichkeit demokratischer Strukturen erhöht. Glaeser et al. (2004) konzentrieren sich in ihrer Arbeit stark auf die Rolle von ökonomischen Institutionen wie Eigentumsrechten und betonen deren Wichtigkeit für die ökonomische Entwicklung. Ihre Evidenz dafür, dass Humankapital die zentrale Determinante von Wirtschaftswachstum ist, während Institutionen in Form von Demokratie eine untergeordnete Rolle im Wachstumsprozess spielen, lässt die Interpretation zu, dass Wohlstand demokratischen Strukturen förderlich ist. Das empirisch robusteste Ergebnis ist jedoch, dass Humankapitalakkumulation selbst unter Diktaturen zur Armutssenkung führt und die dadurch vorangetriebene wirtschaftliche Entwicklung die Verbesserung von politischen Institutionen

\footnotetext{
${ }^{17}$ Siehe bspw. (Lipset, 1959; Huntington, 1991). Bourguignon und Verdier (2000) untersuchen den Bildungskanal in einem theoretischen Modell in dem politische Beteiligung vom Bildungsstand der Wirtschaftssubjekte abhängt. Je ärmer ein Land, desto geringer ist die Chance, Demokratisierung durch das Volk voranzutreiben und damit auch den Wachstumsprozess der Wirtschaft weiter anzukurbeln. Bildung ist in diesem Model also Motor von politischer Beteiligung und somit auch von Demokratie und Wachstum. Bertocchi und Spagat (2004) zeigen in einem theoretischen Modell wie eine politisch motivierte Ausgestaltung des Bildungswesens den Entwicklungspfad einer Volkswirtschaft beeinflussen kann.
} 
vereinfacht. Diese Studie liefert jedoch keine Evidenz für einen direkten Effekt von Wohlstand und Wachstum auf demokratische Strukturen.

Acemoglu et al. (2005a) untersuchen den Zusammenhang zwischen Ausbildung und Demokratie. Sie benutzen Längsschnittsdaten und identifizieren kausale Effekte von Humankapitaldichte auf die Wahrscheinlichkeit demokratischer Strukturen mittels Variation innerhalb eines Landes über die Zeit, anstelle von Variation zwischen einzelnen Ländern. Die Ergebnisse widerlegen die frühere Evidenz für kausale Effekte auf Ebene von Querschnittskorrelationen und zeigen, dass Ausbildung selbst keinen signifikanten Effekt aufweist. Für eine Übersicht der entsprechenden Literatur und der Effekte von Humankapital siehe Tabelle 2.

\subsection{Polit-ökonomische Kanäle}

Einkommensungleichheit. Analog zur Argumentation zuvor erscheinen demokratische Strukturen a priori weniger vorteilhaft für den reicheren Teil der Bevölkerung, da sie erhöhten Umverteilungsdruck implizieren. Gemäß diesem Gedankengang zeigen Bourguignon und Verdier (2000), dass ungleiche oligarchische Gesellschaften später demokratische Reformen implementieren und sich auch langsamer entwickeln als solche, die weniger Ungleichheit aufweisen. Boix und Stokes (2003) sowie Boix (2003) argumentieren, dass nicht der Entwicklungsstand demokratische Strukturen verursacht, sondern andere damit verbundene Veränderungen, wie niedrigere Einkommensungleichheit. Demnach führt wirtschaftliche Entwicklung zum Zusammenbruch von Diktaturen und zur Aufrechterhaltung von Demokratie, solange sie mit geringerer Einkommensungleichheit einhergeht. Geringere Einkommensungleichheit impliziert jedoch, dass der Umfang progressiver Umverteilungsprogramme geringer ausfällt. Dadurch ist der Widerstand der reichen Bevölkerungsschichten gegen demokratische Reformen geringer, und demokratische Reformen sind wahrscheinlicher.

Die Relevanz des „Konsenses der Mitte“ - einer nationalen Situation in der weder starke Klassenunterschiede noch große ethnische Unterschiede existieren - wird von Easterly (2001) als wichtiges Verbindungsstück zwischen Entwicklung und Demokratie gesehen. Die Erklärung knüpft direkt an die Argumentation zu Einkommensungleichheit an. Solch ein Konsens ermöglicht einen höheren Entwicklungsstand durch verbesserte Bedingungen für die Schaffung von Humankapital und Infrastruktur, größere politische Stabilität und Urbanisierung, Faktoren, die wiederum Grundvorrausetzung für eine demokratische Staatsführung sind. Easterly findet Evidenz für einen signifikanten positiven Effekt eines Mittelklassenkonsenses auf Demokratie wie auch auf Wachstum. 
Politikmaßnahmen und Handel. Handelsoffenheit bietet eine weitere Möglichkeit für den Einfluss wirtschaftlicher Entwicklung auf politische Strukturen. Wirtschaftlich entwickelte Länder nutzen komparative (Kosten-)Vorteile sowie eine hochwertige Infrastruktur und können auf diese Weise vom internationalen Handel profitieren. Umgekehrt werden dadurch Demokratisierungsprozesse angestoßen oder bereits etablierte demokratische Strukturen stabilisiert. Rigobon und Rodrik (2004) untersuchen die Zusammenhänge zwischen politischen Institutionen, Offenheit und wirtschaftlicher Entwicklung. Ihre empirischen Ergebnisse zeigen allerdings, dass Entwicklung nur mäßig gut für Offenheit ist, und dass Offenheit einen negativen Einfluss auf das Auftreten von Demokratie hat. Eine mögliche Interpretation dieser Ergebnisse ist, dass durch Handelsoffenheit Verteilungskonflikte verschärft werden, die eine Demokratie belasten und demokratische Reformen erschweren.

Natürliche Ressourcen und historische Faktoren. Ein großer Teil der Literatur, die sich mit der Demokratie-Entwicklungs-Frage beschäftigt, untersucht die Auswirkung des Vorhandenseins von natürlichen Ressourcen auf den Demokratisierungsprozess. Dabei besteht weitgehend die Überzeugung, dass ein reicher Schatz an natürlichen Ressourcen sich negativ auf Demokratie auswirkt. Dies hat vor allem mit der damit verbundenen Ungleichheit zu tun, die sich durch die ungleiche Verteilung der Bodenschätze zugunsten der Eliten manifestiert. Barro (1999) findet hierfür empirische Evidenz.

Eng verbunden mit den Effekten des Ressourcenreichtums eines Landes ist die Kolonialisierung von besonders ertragreichen Gebieten und Regionen. Die Kolonialmächte des 19. Jahrhunderts haben ihre jeweiligen Kolonien nicht nur ausgebeutet sondern ihnen insbesondere wenn die Lebensumstände eine dauerhafte Besiedlung durch Kolonialherren ermöglichten - auch ihre Institutionen aufgezwungen (Acemoglu et al., 2001). Diese Institutionen beeinflussten wiederum die Entwicklung von politischen Institutionen in diesen Kolonien, selbst nach dem Ende der Kolonialzeit. Barro (1999) findet zwar, dass die Kolonialgeschichte eines Landes keinen direkten signifikanten Einfluss auf die Demokratie eines Landes hat, und argumentiert, dass es stattdessen einen indirekten Effekt über den Lebensstandard im Land geben könnte. Aber die Ergebnisse von Acemoglu et al. (2001) unter Verwendung von Daten über die Mortalität von Kolonialherren in den Kolonien als Instrument für die Qualität von ökonomischen Institutionen sprechen für einen Einfluss durch die Kolonialgeschichte und die daraus resultierenden institutionellen Rahmenbedingungen. Ressourcenreichtum und der daraus folgende Kolonialismus hat zudem die Einkommenskluft zwischen den Arm und Reich und dadurch den Wachstumspfad der betroffenen Länder beeinflusst, wie durch die Evidenz von Gylfason (2001) und Galor et al. (2005) gezeigt wird. Aus einer ähnlichen Sicht diskutieren Engerman und Sokoloff (2005) die Geschichte ehemaliger 
Kolonien in Amerika. Ihren Ergebnissen zufolge haben Kolonien mit großer anfänglicher Ungleichheit in der Ressourcenverteilung oder einem geographischen Umfeld, das Großgrundbesitz förderte, sowie mit heterogener Bevölkerung, generell dazu tendiert Institutionen $\mathrm{zu}$ entwickeln, die den Zugang zu wirtschaftlichen Möglichkeiten einschränkten und wenig in Schulen und anderer Infrastruktur investierten. Im Nachhinein stellte sich dies als entscheidendes Hindernis für eine nachhaltige wirtschaftliche Entwicklung, aber auch für eine funktionierende Demokratie heraus.

Urbanisierung. Der Effekt von Verstädterung auf die politische Struktur ist a priori ambivalent. Auf der einen Seite hat eine ländliche Bevölkerung nur eingeschränkte Möglichkeiten sich zu organisieren, was es Diktatoren vereinfacht, ihre Macht zu erhalten. Andererseits ist die Überwachung dünn besiedelter Gebiete für die zentrale Autorität schwieriger. Barro (1999) findet einen signifikant negativen Effekt von Urbanisierung auf das Auftreten von demokratischen Strukturen.

Zusammenfassend lässt sich sagen, dass die Evidenz für einen direkten Einfluss von wirtschaftlichem Wohlstand auf Demokratie nicht sehr stark ist. Das Vorherrschen demokratischer Strukturen scheint vielmehr hauptsächlich von der Einkommens- und Ressourcenverteilung beeinflusst zu werden, wie auch nochmals aus der Zusammenstellung in Tabelle 2 hervorgeht.

\section{Zusammenfassung und Ausblick}

Der Überblick über die empirische Literatur zur Frage nach den kausalen Zusammenhängen hinter der positiven Korrelation zwischen wirtschaftlichem Wohlstand und Demokratie zeigt ein Bild komplexer Zusammenhänge. Der Entwicklungsgrad einer Volkswirtschaft per se scheint die Wahrscheinlichkeit demokratischer Strukturen nicht zu erhöhen. Dies geschieht höchstens indirekt, wie beispielsweise durch geringere Einkommensunterschiede und ein hohes Bildungsniveau in der Bevölkerung. Auf der anderen Seite entfalten demokratische Strukturen offensichtlich in der Tat, vor allem mittels günstiger Bedingungen für Humankapitalakkumulation, positive Effekte auf den Entwicklungsstand und die Wachstumsrate. Am wichtigsten für die Entwicklung eines Landes scheinen in diesem Zusammenhang jedoch ökonomische Institutionen, insbesondere Rechtsstaatlichkeit, die Gewährleistung von Eigentumsrechten und die effektive Bekämpfung von Korruption zu sein. Demokratie hat demnach einen wachstumsförderlichen Effekt soweit sie diese Institutionen effektiv implementiert. Die Frage, ob demokratische Strukturen ursächlich, oder gar notwendig für die Implementierung solcher förderlichen ökonomischen Institutionen sind, bleibt auf Basis der verfügbaren Evidenz letztlich offen. Wie so oft dürfte der Schlüssel zur Antwort im Detail und 
den Interaktionen unterschiedlicher institutioneller Dimensionen liegen, wie auch neue Ergebnisse von Persson und Tabellini (2006) belegen.

Vor diesem Hintergrund gibt es Bestrebungen in der neueren Literatur, die Ausgestaltung von institutionellen Strukturen, sowie ihre Determinanten und Effekte, im Detail zu untersuchen, anstelle sich lediglich auf deren Existenz, wie etwa das Vorhandensein demokratischer Strukturen, zu beschränken. Feld und Savioz (1997) zeigen etwa, dass direkte demokratische Strukturen wie Volksentscheide zu effizienteren Politikmaßnahmen führen. Persson und Tabellini (2004) und Persson (2005) zeigen, dass Präsidialsysteme und Mehrheitswahlsysteme typischerweise mit kleineren Staaten assoziiert sind. Acemoglu und Johnson (2005) öffnen die black box der ökonomischen Institutionen, und beleuchten die Unterschiede zwischen Institutionen, die Eigentumsrechte gegenüber dem Staat schützen, und Institutionen zum Schutz von Eigentumsrechten im privatrechtlichen Zusammenhang. Ganz im Sinne Hayeks zeigen sie, dass primär die erstgenannten Institutionen relevant für die wirtschaftliche Entwicklung eines Landes sind. Eine weitere offene Frage ist, unter welchen Umständen stabile Demokratien implementiert werden können. Erste Schritte die dynamischen Interaktionen zwischen politischen und ökonomischen Institutionen $\mathrm{zu}$ modellieren werden etwa in Acemoglu et al. (2005c) oder Cervellati et al. $(2005,2006)$ gemacht, doch diese Fragen werden die Forschung noch einige Zeit beschäftigen.

Obwohl Demokratie sowohl aus humanitären und philosophischen, aber auch aus ökonomischen Gründen sehr wohl erstrebenswert scheint, so ist vor dem Hintergrund der beschriebenen Evidenz zu bezweifeln, ob die unter Umständen gewaltsame und von externen Mächten initiierte Implementierung demokratischer Strukturen ausreicht, um in unterentwickelten Ländern einen Wachstums- und Konvergenzprozess in Gang zu setzen. Ganz zu schweigen von der Frage, ob sich diese Demokratien als ausreichend stabil erweisen, um wirksame Institutionen und Politikmaßnahmen erfolgreich $\mathrm{zu}$ implementieren. Die aktuelle Entwicklung im Nahen Osten zeigt jedenfalls, dass Demokratie weder leicht zu implementieren ist, noch, dass sie, einmal implementiert, Bestand hat und automatisch wirtschaftliche Entwicklungsprozesse in Gang setzt. Ziel der Forschung muss daher sein, noch mehr über die Wechselwirkungen zwischen ökonomischen und politischen Institutionen und ihren Determinanten herauszufinden. 


\section{Literaturverzeichnis}

Acemoglu, D. und Robinson, J. A. (2000), Why Did The West Extend The Franchise? Democracy, Inequality, And Growth In Historical Perspective, The Quarterly Journal of Economics 115(4), 1167-1199.

Acemoglu, D., Johnson, S., Robinson, J. (2001), The Colonial Origins of Comparative Development: An Empirical Investigation, American Economic Review 91(5), 1369-1401.

Acemoglu, D., Johnson, S., Robinson, J. (2002), Reversal of Fortune: Geography and Institutions in the Making of the Modern World Income Distribution, Quarterly Journal of Economics 117(4), 1231-1294.

Acemoglu, D. und Robinson, J. A. (2003), A Theory of Political Development, mimeo, MIT.

Acemoglu, D., Johnson, S., Robinson, J. und Yared, P. (2005a), From Education to Democracy?, American Economic Review 95(2), 44-49.

Acemoglu, D., Johnson, S., Robinson, J. und Yared, P. (2005b), Income and Democracy, NBER Working Papers 11205.

Acemoglu, D., Johnson S. und Robinson J. (2005c), Institutions as the Fundamental Cause of Long-Run Growth, in: Aghion u. Durlauf (Hrsg.), Handbook of Economic Growth, Vol. 1A, Kap. 6.

Ades, A. und DiTella, R. (1999), Rents, Competition, and Corruption, American Economic Review 89(4), 982-993.

Barro, R. J. (1991). "Economic Growth in a Cross Section of Countries," NBER Working Papers 3120, National Bureau of Economic Research, Inc.

Barro, R. J. (1999). "Determinants of Democracy,” Journal of Political Economy, University of Chicago Press, vol. 107(S6), pages S158-29.

Barro, R. J. (2000), Rule of Law, Democracy, and Economic Performance, in: M. A. Miles et al. (Hrsg.), 2000 Index of Economic Freedom. The Heritage Foundation, Washington, D.C.

Bertocchi, G. und Spagat, M. (2004), The Evolution of Modern Educational Systems: Technical Vs. General Education, Distributional Conflict and Growth, Journal of Development Economics 73(2), 559-582.

Boix, C. (2003), Democracy and Redistribution, Cambridge University Press, Cambridge.

Boix, C. und Stokes, S. C. (2003). "Endogenous Democratization,” World Politics 55, No. 4, 148.

Bourguignon, F. und Verdier, T. (2000), Oligarchy, democracy, inequality and growth, Journal of Development Economics 62(2), 285-313.

Burkhart, R. und Lewis-Beck, M. (1994), Comparative Democracy: The Economic Development Thesis, American Political Science Review 88, 903-910. 
Cervellati, M., Fortunato, P. und Sunde, U. (2005), Hobbes to Rousseau: Inequality, Institutions and Development, IZA Discussion Paper 1450.

Cervellati, M., Fortunato, P. und Sunde, U. (2006), Consensual and Conflictual Democratization, IZA Discussion Paper 2225.

Chowdhury, S.K. (2004), Do Democracy and Press Freedom Reduce Corruption? Evidence From a Cross-Country Study, ZEF Discussion Papers on Development Policy 85, Center for Development Research, Bonn.

Dahl, R. A. (1971), Polyarchy, Participation and Opposition, Yale University Press, New Haven.

Dabla-Norris, E. und Gradstein, M. (2004), The Distributional Bias of public education: causes and consequences, IMF Working Paper 04/214.

Dollar, D., und Kraay, A. (2003), Institutions, Trade and Growth, Journal of Monetary Economics 50(1), 133-162.

Easterly, W. (2001), The Middle Class Consensus and Economic Development, Journal of Economic Growth 6(4), 317-335.

Easterly, W. (2005), National Policies and Economic Growth: A Rappraisal, in: Aghion u. Durlauf (Hrsg.), Handbook of Economic Growth, Vol. 1A, Kap. 15.

Engerman, S. L. und Sokoloff, K. L. (2002), Factor Endowments, Inequality and Paths of Development Among New World Economies, NBER Working Papers 9259.

Engerman, S. L. und Sokoloff, K. L. (2005), Colonialism, Inequality, and Long-Run Paths of Development, NBER Working Papers 11057.

Feld, L.P. und Savioz, M.R. (1997), Direct Democracy Matters for Economic Performance: An Empirical Investigation, Kyklos 50(4), 507-538.

Feld, L.P. und Voigt, S. (2003), Economic Growth and Judicial Independence: Cross-Country Evidence Using a New Set of Indicators, European Journal of Political Economy 19(3), 497527.

Galor, O., Moav, O. und Vollrath, D. (2005), Land Inequality and the Emergence of Human Capital Promoting Institutions, Brown University Economics Working Paper 05-03.

Glaeser, E. L., La Porta, R., Lopez-de-Silanes, F. und Shleifer, A. (2004), Do Institutions Cause Growth?, Journal of Economic Growth 9(3), 271-303.

Gylfason, T. (2001), Natural resources, education, and economic development, European Economic Review 45(4-6), 847-859.

Hall, R. E. und Jones, C. I. (1999), Why Do Some Countries Produce So Much More Output Per Worker Than Others?, The Quarterly Journal of Economics 114(1), 83-116.

Harrington, J. 1656 (1883), The Commonwealth of Oceania. Henry Morley, London. 
Hayek, F.A. (2005), Die Verfassung der Freiheit, Mohr Siebeck, 4. durchges. Aufl.

Heitger, B. (2004), Property Rights and the Wealth of Nations: A Cross-Country Study, Cato Journal 23 (3), 381-402.

Hobbes, T. (1651), The Leviathan,

(http://etext.library.adelaide.edu.au/h/hobbes/thomas/h681/h681.html)

Huntington, S. (1991), The Third Wave: Democratization in the Late Twentieth Century. University of Oklahoma Press, London.

Knack, S. und Keefer, P. (1995), Institutions and Economic Performance: Cross-Country Tests Using Alternative Institutional Measures, Economics and Politics 7, 207-227.

Kurzman, C., Werum, R. und Burkhart, R. E. (2002), Democracy's Effect on Economic Growth:

A Pooled Time-Series Analysis, 1951-1980, Studies in Comparative International Development 37 (1), 3-33.

Lederman, D., N.V. Loyaza und R. Soares (2005), Accountability and Corruption: Political Institutions Matter, Economics and Politics 17(1), 1-35.

Lijphart, A. (1999), Patterns of Democracies. Yale University Press, New Haven.

Lipset, S. M. (1959), Some social requisites of democracy: economic development and political legitimacy, The American Political Science Review 53(3), 69-105.

Lipset, S. M. (1960), Political Man: the Social Bases of Politics. Doubleday, Garden City, N.Y. Lizzeri, A. und Persico, N. (2004), Why Did the Elites Extend the Suffrage? Democracy and the Scope of Government, With an Application to Britain's "Age of Reform", The Quarterly Journal of Economics 119(2), 705-763.

Lott, J. R., Jr. (1999), Public Schooling, Indoctrination, and Totalitarianism, Journal of Political Economy 107(6), 127-129.

Lucas, R.E. jr. (1988), On the Mechanics of Economic Development, Journal of Monetary Economics 22(1), 3-42.

Mauro, P. (1995), Corruption and Growth, Quarterly Journal of Economics 110(3), 681-712.

Mazo, E. (2005), What Causes Democracy?, CDDRL Working Paper 28.

Meltzer, A. H. und Richard, S. F. (1981), A Rational Theory of the Size of Government, Journal of Political Economy 89, 914-927.

Minier, J. A. (1998), Democracy and Growth: Alternative Approaches, Journal of Economic Growth 3(3), 241-266.

Mobarak, A. M. (2005), Democracy, Volatility, and Economic Development, Review of Economics and Statistics 87(2), 348-361.

Mulligan, C., R. Gil und X. Sala-i-Martin (2004), Do Democracies Have Different Public Policies?, Journal of Economic Perspectives 18(1), 51-74. 
Muno, W. (2001), Demokratie und Entwicklung, Dokumente und Materialien 29, Politikwissenschaft, Universität Mainz.

Niskanen, W. (1971), Bureaucracies and Representative Government, Aldine Atherton, Chicago.

North, D. C. (1990), Institutions, Institutional Change and Economic Performance. Cambridge UP, Cambridge.

Olson, M. (1982), The Rise and Decline of Nations, Yale University Press, New Haven.

Olson, M. (1993), Dictatorship, Democracy, and Development, American Political Science Review, 87, 567-576.

Papaioannou, E. und Siourounis, G. (2004), Democratization and Growth, London Business School Economics Working Paper.

Perotti, R. (1996), Growth, Income Distribution, and Democracy: What the Data Say, Journal of Economic Growth 1(2), 149-187.

Persson, T. (2005), Forms of Democracy, Policy, and Economic Development, CEPR Discussion Paper 4938.

Persson, T. und Tabellini, G. (1994), Is Inequality Harmful for Growth?, American Economic Review 84(3), 600-621.

Persson, T. und Tabellini, G. (2004), Constitutional Rules and Fiscal Policy Outocomes, American Economic Review 94(1), 25-45.

Persson, T. und Tabellini, G. (2006), Democracy and Development: The Devil in the Details, American Economic Review 96(2), im Erscheinen.

Pettersson, J. (2004), Democracy, Regime Stability, and Growth, Research Papers in Economics, Stockholm University.

Przeworski, A. (2004), The Last Instance: Are Institutions a Deeper Cause of Economic

Development?, European Archives of Sociology 45(2), 165-188.

Przeworski, A. und Limongi, F. (1997), Modernization. Theories and Facts, World Politics 1, $155-183$.

Rigobon, R. und Rodrik, D. (2004), Rule of Law, Democracy, Openness and Income: Estimating the Interrelationships, CEPR Discussion Papers 4653.

Rodrik, D., Subramanian, A. und Trebbi, F. (2004, Institutions Rule: The Primacy of Institutions Over Geography and Integration in Economic Development, Journal of Economic Growth 9(2), 131-165.

Rodrick, D. und Wacziarg, R. (2005), Do Democratic Transitions Produce Bad Economic Outcomes?, CDDRL Working Papers 29.

Sachs, J. und Warner, A. (1995), Economic Reform and the Process of Global Integration, Brookings Papers on Economic Activity 1, 1-95. 
Schneider, F. (2004), The Size of the Shadow Economies in 145 Countries all over the World: First Results over the Period 1999 to 2003, IZA Discussion Paper 1431.

Sala-i-Martin, X. (1997), I Just Ran Two Million Regressions, American Economic Review 87(2), 178-183.

Solow, R. (1956), A Contribution to the Theory of Econoimc Growth, Quarterly Journal of Econonomics 70(1), 65-94.

Tavares, J. und Wacziarg, R. (2001), How Democracy Affects Growth, European Economic Review 45(8), 1341-1378.

Treisman, D. (2000), The Causes of Corruption: A Cross-National Study, Journal of Public Economics 76(3), 399-457.

Wacziarg, R. (2001), Human Capital and Democracy, unpublished manuscript, Stanford Business School, January 2001.

Wu, W. und Davis, O. A. (1999), The Two Freedoms, Economic Growth and Development: An Empirical Study, Public Choice 100(1-2), 39-64. 
Tabelle 1: Institutioneller Ansatz, Effekt von Demokratie auf Wirtschaftswachstum

\begin{tabular}{|c|c|c|c|c|}
\hline Untersuchter Effekt/Kanal & Studie & Daten & Schätzmethode & Effekt \\
\hline \multicolumn{5}{|c|}{ Burkhart und Lewis-Beck (1994) } \\
\hline & Burkhart und Lewis-Beck (1994) & Panel (131 Länder, 1972-1989) & GLS-ARMA & 0 \\
\hline & Sala-i-Martin (1997) & Querschnitt & OLS & + \\
\hline & Papaioannou und Siourounis (2004) & Panel (67 Länder, 1960-2000) & OLS, IV, Fixed Effects,GMM & + \\
\hline & Pettersson (2004) & Panel (103 Länder, 1961-2000) & OLS & + \\
\hline & Rigobon und Rodrik (2004) & 43 Länder in jeder Unterstichprobe & Identification through Heteroskedasticity & + \\
\hline & Rodrik und Wacziarg (2005) & Panel (74/154 Länder, 1950-2000) & OLS, Fixed Effects & + \\
\hline & Tavares und Wacziarg (2001) & Panel (65 Länder, 1970-1989) & 3SLS & - \\
\hline & Wu und Davis (1999) & Panel (100 Länder, 1975-1992) & OLS, Fixed Effects & 0 \\
\hline & Barro (2000) & Panel (138 Länder, 1972-1998) & OLS, IV & 0 \\
\hline & Glaeser et al. (2004) & Panel (102 Länder, 1969-2000) & OLS, Fixed Effects & 0 \\
\hline \multicolumn{5}{|l|}{ ökonomische Kanäle } \\
\hline Kapital & Minier (1998) & Panel (131 Länder, 1965-1989) & Regression Tree Analysis & + \\
\hline & Tavares und Warcziarg (2001) & Panel (65 Länder, 1970-1989) & 3SLS & - \\
\hline Humankapital & Galor, Moav und Vollrath (2005) & Panel (US Staaten, 1880-1920) & 2SLS & + \\
\hline & Lott $(1999) *$ & Querschnitt (99 Länder, 1985-1992) & OLS & $-*$ \\
\hline & Minier (1998) & Panel (131 Länder, 1965-1989) & Regression Tree Analysis & + \\
\hline & Tavares und Wacziarg (2001) & Panel (65 Länder, 1970-1989) & 3SLS & + \\
\hline & Wacziarg (2001) & Panel (1970-1990) & OLS, Seemingly Unrelated Regression & + \\
\hline \multicolumn{5}{|l|}{ polit-ökonomische Kanäle } \\
\hline \multirow[t]{3}{*}{ Einkommensungleichheit } & Persson und Tabellini (1994) & Panel (9/56 Länder, 1830-1985/1960-85) & OLS & + \\
\hline & Tavares und Warcziarg (2001) & Panel (65 Länder, 1970-1989) & 3SLS & + \\
\hline & Perotti (1996) & Querschnitt (65 Länder, 1960-1985) & OLS, 2SLS & 0 \\
\hline \multirow{3}{*}{ Staatskonsum, Steuern } & Tavares und Warcziarg (2001) & Panel (65 Länder, 1970-1989) & 3SLS & - \\
\hline & Papaioannou und Siourounis (2004) & Panel (67 Länder, 1960-2000) & OLS, IV, Fixed Effects,GMM & - \\
\hline & Mulligan et al. (2004) & Querschnitt (131 Länder) & OLS & 0 \\
\hline \multirow[t]{2}{*}{ Handelsoffenheit } & Tavares und Warcziarg (2001) & Panel (65 Länder, 1970-1989) & 3SLS & 0 \\
\hline & Papaioannou und Siourounis (2004) & Panel (67 Länder, 1960-2000) & OLS, IV, Fixed Effects,GMM & + \\
\hline \multirow[t]{4}{*}{ Instabilität } & Perotti (1996) & Querschnitt (65 Länder, 1960-1985) & OLS, 2SLS & 0 \\
\hline & Tavares und Warcziarg (2001) & Panel (65 Länder, 1970-1989) & 3SLS & 0 \\
\hline & Treismann (2000) & Panel (64 Länder, 1980-1998) & OLS, WLS & + \\
\hline & Mobarak (2005) & Panel (136/77 Länder, 1960-2000) & OLS, IV, 2SLS, 3SLS, Random Effects & \\
\hline \multicolumn{5}{|l|}{ ökonomische Institutionen } \\
\hline \multirow[t]{11}{*}{ Eigentumsrechte/Rechtsstaat } & Knack und Keefer (1995) & Querschnitt (97 Länder) & OLS & + \\
\hline & Sala-i-Martin (1997) & Querschnitt & OLS & + \\
\hline & Wu und Davis (1999) & Panel (102 Länder, 1975-1990) & OLS & + \\
\hline & Barro (2000) & Panel (114 Länder, 1982-1999) & IV & + \\
\hline & Acemoglu et al. (2001)** & Querschnitt (64 Länder) & OLS, 2SLS & $+* *$ \\
\hline & Acemoglu et al. (2002)** & Querschnitt (162 Länder)/Panel (55 Länder) & OLS & $+* *$ \\
\hline & Feld und Voigt (2003) & Querschnitt (71 Länder) & OLS & + \\
\hline & Heitger $(2004)^{* *}$ & Panel (84 Länder, 1975-1995) & OLS, 2SLS, IV & $+* *$ \\
\hline & Rigobon und Rodrik (2004) & 43 Länder in jeder Unterstichprobe & Identification through Heteroskedasticity & + \\
\hline & Rodrik et al. (2004) & Querschnitt (137 Länder) & OLS, IV & + \\
\hline & Glaeser et al. (2004) & Panel (102 Länder, 1969-2000) & OLS, Fixed Effects & 0 \\
\hline \multirow[t]{4}{*}{ Korruption } & Ades und di Tella (1999) & Panel (31/52 Länder, 1980-1990) & OLS, 2SLS, IV & $0 /+$ \\
\hline & Treismann (2000) & Panel (64 Länder, 1980-1998) & OLS, WLS & + \\
\hline & Chowdhury (2004) & Panel (97 Länder, 1995-2002) & OLS, IV, Fixed and Random Effects, GMM & + \\
\hline & Ledermann et al. (2005) & Panel (179 Länder, 1984-1999) & OLS, Fixed Effects, Ordered Probit & + \\
\hline
\end{tabular}


Tabelle 2: Entwicklungsansatz, Effekt von Entwicklungsstand auf Demokratie

\begin{tabular}{|c|c|c|c|c|}
\hline Untersuchter Effekt/Kanal & Studie & Daten & Schätzmethode & Effekt \\
\hline \multicolumn{5}{|l|}{ direkt } \\
\hline & Burkhart und Lewis-Beck (1994) & Panel (131 Länder, 1972-1989) & GLS-ARMA & + \\
\hline & Przeworski und Limongi (1997) & Panel (135 Länder, 1950-1990) & OLS & 0 \\
\hline & Barro (1999) & Panel (100 Länder, 1960-1995) & OLS & + \\
\hline & Wu and Davis (1999) & Panel (100 Länder, 1975-1992) & OLS & + \\
\hline & Acemoglu und Robinson (2003) & Panel (1965-2000) & OLS, Fixed Effects & 0 \\
\hline & Boix und Stokes (2003) & Panel (135 Länder) & Dynamic Probit & + \\
\hline & Acemoglu et al. (2005b) & Panel (1840-2000) & IV, Fixed Effects & 0 \\
\hline & Glaeser et al. (2004) & Panel (102 Länder, 1969-2000) & OLS, Fixed Effects & 0 \\
\hline \multicolumn{5}{|l|}{ ökonomische Kanäle } \\
\hline \multirow[t]{3}{*}{ Humankapital } & Barro (1999) & Panel (100 Länder, 1960-1995) & & \\
\hline & Glaeser et al. (2004) & Panel (102 Länder, 1969-2000) & OLS, Fixed Effects & 0 \\
\hline & Acemoglu et al. (2005a) & Panel (108 Länder, 1965-2000) & OLS, GMM & 0 \\
\hline \multicolumn{5}{|l|}{ polit-ökonomische Kanäle } \\
\hline \multirow[t]{3}{*}{ Niedrigere Ungleichheit } & Boix and Stokes (2003) & Panel (135 Länder ) & OLS & + \\
\hline & Boix (2003) & Panel (>50 Länder, 1950-1990) & OLS & + \\
\hline & Easterly (2001) & Querschnitt (197 Länder) & 2SLS, 3SLS, Probit & + \\
\hline Handelsoffenheit & Rigobon und Rodrik (2004) & 43 Länder in jeder Unterstichprobe & Identification through Heteroskedasticity & - \\
\hline \multirow[t]{3}{*}{ Natürliche Ressourcen } & Barro (1999) & Panel (100 Länder, 1960-1995) & OLS & - \\
\hline & Acemoglu et al. (2001) & Querschnitt (64 Länder) & OLS, IV & - \\
\hline & Galor, Moav undVollrath (2005) & Panel (US Staaten, 1880-1920) & 2SLS & - \\
\hline Kolonialisierung & Barro (1999) & Panel (100 Länder, 1960-1995) & OLS & 0 \\
\hline Urbanisierung & Barro (1999) & Panel (100 Länder, 1960-1995) & OLS & - \\
\hline
\end{tabular}

